\title{
The feasibility of local anesthesia for the surgical treatment of umbilical hernia: a systematic review of the literature
}

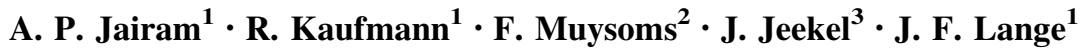

Received: 22 July 2016/ Accepted: 6 January 2017/Published online: 20 January 2017

(C) The Author(s) 2017. This article is published with open access at Springerlink.com

\begin{abstract}
Background Yearly approximately 4500 umbilical hernias are repaired in The Netherlands, mostly under general anesthesia. The use of local anesthesia has shown several advantages in groin hernia surgery. Local anesthesia might be useful in the treatment of umbilical hernia as well. However, convincing evidence is lacking. We have conducted a systematic review on safety, feasibility, and advantages of local anesthesia for umbilical hernia repair. Methods A systematic review was conducted according to the PRISMA guidelines. Outcome parameters were duration of surgery, surgical site infection, perioperative and postoperative complications, postoperative pain, hernia recurrence, time before discharge, and patient satisfaction. Results The systematic review resulted in nine included articles. Various anesthetic agents were used, varying from
\end{abstract}

A. P. Jairam

a.jairam@erasmusmc.nl

R. Kaufmann

r.kaufmann@erasmusmc.nl

F. Muysoms

filip.muysoms@gmail.com

J. Jeekel

j.jeekel@erasmusmc.nl

J. F. Lange

j.lange@erasmusmc.nl

1 Department of Surgery, Erasmus University Medical Center Rotterdam, Room Z-835, 3000 CA Rotterdam, The Netherlands

2 Department of Surgery, Algemeen Ziekenhuis Maria Middelares, Kortrijksesteenweg 1026, 9000 Ghent, Belgium

3 Department of Neuroscience, Erasmus University Medical Center, Rotterdam, 3000 CA Rotterdam, The Netherlands short acting to longer acting agents. There was no consensus regarding the injection technique and no conversions to general anesthesia were described. The most common postoperative complication was surgical site infection, with an overall percentage of $3.4 \%$. There were no postoperative deaths and no allergic reactions described for local anesthesia. The hernia recurrence rate varied from 2 to $7.4 \%$. Almost $90 \%$ of umbilical hernia patients treated with local anesthesia were discharged within $24 \mathrm{~h}$, compared with $47 \%$ of patients treated with general anesthesia. The overall patient satisfaction rate varied from 89 to $97 \%$. Conclusion Local anesthesia for umbilical hernia seems safe and feasible. However, the advantages of local anesthesia are not sufficiently demonstrated, due to the heterogeneity of included studies. We, therefore, propose a randomized controlled trial comparing general versus local anesthesia for umbilical hernia repair.

Keywords Umbilical hernia - Local anesthesia · Systematic review

\section{Introduction}

Umbilical hernia is a common diagnosis in surgery $[1,2]$. Approximately, $10 \%$ of all abdominal wall hernias are defined as umbilical hernia [3], and the prevalence of umbilical hernia in the adult population is $2 \%$ [4]. The European Hernia Society defines a primary umbilical hernia as a ventral hernia present at birth or developed spontaneously without trauma to the abdominal wall as the cause of the hernia and with its center at the umbilicus [5]. Each year, approximately 4500 umbilical hernias are repaired in the Netherlands and most of these patients are operated under general anesthesia. 
Worldwide, ever more patients undergo ambulatory hernia surgery performed under local anesthesia [6]. Local anesthesia in the treatment for groin hernias has been already thoroughly investigated. Studies showed the superiority of local anesthesia for open groin hernia repair than general anesthesia or spinal anesthesia [7-13]. However, only $7 \%$ of Dutch surgeons uses local anesthesia in Lichtenstein repair [13]. This is surprising, since the use of local anesthesia could prevent complications related to general anesthesia. Possible advantages of the use of local anesthesia are less postoperative pain and extended postoperative analgesia, less perioperative and postoperative complications, early mobilization and, therefore, a shorter duration of hospital stay. Furthermore, use of local anesthesia could be more cost-effective than general anesthesia or spinal anesthesia, since there is no anesthesiologist needed and only less expensive local anesthetics are used [7, 13-16]. There is a lack of convincing literature on umbilical hernia repaired under local anesthesia [1]. We have conducted a systematic review of the literature on the safety, feasibility, and advantages of local anesthesia for the repair of umbilical hernia.

\section{Materials and methods}

We conducted a systematic review following the PRISMA guidelines [17]. A systematic search was performed in MEDLINE, Embase, Web of Science, Scopus, PubMed Publisher, and the Cochrane Library.

The search strategy was prepared by the Biomedical Information Specialist of the Medical Library (Erasmus University Medical Center, Rotterdam, the Netherlands). A syntax with search terms was designed, which is available at Appendix 1.

Records identified were independently evaluated by two reviewers. All records were screened by title and abstract for eligibility, and the full text of eligible records was assessed. Studies were included into the analysis if they met the following inclusion criteria: adult patients with umbilical hernia or paraumbilical hernia, who were operated under local anesthesia with or without a control group operated with another type of anesthesia. Articles had to be written in Dutch, English or German, and randomized controlled trials, cohort studies and case series (with more than 5 patients) were included. Exclusion criteria were studies investigating local anesthesia for other types of hernia than umbilical hernias, laparoscopic surgery, and animal studies or in vitro experiments.

The following outcome measurements were assessed: postoperative pain, duration of surgery, surgical site infection, perioperative and postoperative complications, hernia recurrence, time before discharge, and patient satisfaction. We also extracted the baseline study characteristics from all included studies: study design, study period, and year of publication. The quality of the studies was assessed on the Level of Evidence scale of the Oxford Centre for Evidence-based Medicine [18].

Both reviewers independently sampled the data in a standardized database. This database was set up in Microsoft Office Excel 2010. The data presented in this review were directly abstracted from the original articles. No statistical analyses were performed.

\section{Results}

A total of 1107 articles were identified after the removal of duplicates. After screening of these records 77 articles were found eligible for further assessment. After assessment of the full text versions of these 77 articles, 9 articles were suitable for inclusion in this review. The reasons for exclusion were as follows: anesthesia or umbilical hernia were not well described and not the main subject, research was performed in children or animals, the article contained a case report, there was only an abstract available, or the article was written in another language than Dutch, English or German. The PRISMA flow diagram is shown in Fig. 1.

Of the nine included articles, six were prospective cohort studies, and three were retrospective cohort studies. No randomized study comparing local versus general anesthesia was found. All studies contained a Level of Evidence of $2 \mathrm{~B}$ on the scale of the Oxford Centre for Evidence-based Medicine. Table 1 gives an overview of the articles we included for this review.

In this review, the following outcome parameters will be highlighted: anesthesia technique, postoperative pain, duration of surgery, surgical site infection, perioperative and postoperative complications, recurrence, time before discharge and patient satisfaction. The anesthesia technique was described to outline if there was any consensus regarding the injection technique and the type of anesthetics.

\section{Surgical technique}

There were two studies in which a Mayo repair was performed, with the classical 'vest over pants' technique $[19,20]$. Bennett et al. inserted a polypropylene soft mesh plug if the defect was $<2 \mathrm{~cm}$. In case the defect was $>2 \mathrm{~cm}$, a preperitoneal pocket was made and a polypropylene soft mesh was placed, with a $2 \mathrm{~cm}$ margin [14]. In the study of Kurzer et al. a cone polypropylene mesh was used for defects $<3 \mathrm{~cm}$, and a flat piece mesh for defects $>3 \mathrm{~cm} \mathrm{[2].} \mathrm{Garcia} \mathrm{et} \mathrm{al.} \mathrm{used} 1 \mathrm{~cm}$ as a cutoff point for a primary suture, and 'large' hernias, as they stated, 
Fig. 1 Flow diagram search

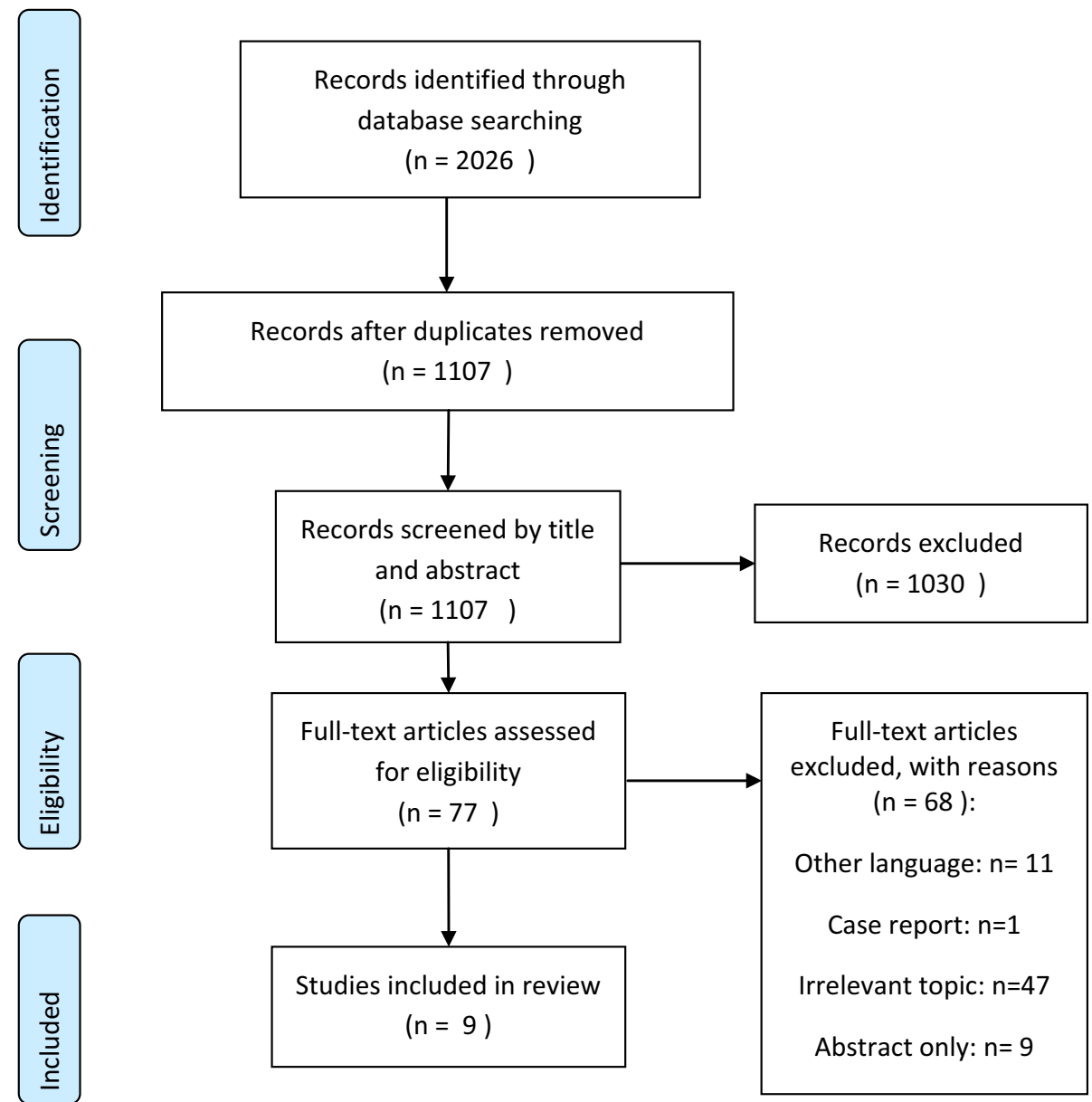

received a polypropylene mesh [4]. Three articles did not mention which cutoff point they used to determine the use of primary sutures or a mesh, and in only one study umbilical hernia operations with meshes was performed $[1,3,6,19]$. Dalenbäck et al. were the only authors who specified the type of surgical procedure for the type of anesthesia. A total of 162 patients underwent an umbilical hernia operation. Of the patients operated with a suture repair, 59\% were operated under local anesthesia and $41 \%$ under general anesthesia. Of the patients receiving a mesh repair, $18 \%$ were operated under local anesthesia and $82 \%$ under general anesthesia.

\section{Anesthesia technique}

There are various anesthesia techniques assessed in the studies. Only Acevedo and Léon described the use of local anesthesia without addition of a sedative [6]. Four other studies combined the use of local anesthesia with sedatives and another four studies used local anesthesia (without sedatives) or general anesthesia for their patient groups $[1-4,14,19-21]$. None of the authors randomized between local anesthesia and general anesthesia. Table 2 shows the various types of anesthesia (local anesthesia or general anesthesia, local anesthesia with our without sedatives) and the different types of anesthetic drugs that were used. The anesthetic drugs varied from the short acting lidocaine and xylocaine to the longer acting agent bupivacaine. Bennett et al. were the only authors who described the injection technique, which was a field block technique: infiltration of the skin and rectus sheath around the umbilicus [14]. Kulacoglu et al. studied patients with umbilical hernia treated with local anesthesia. They stated there were no conversions to general anesthesia; all patients tolerated local anesthesia and there were no intraoperative anesthesia-related complications [1].

\section{Postoperative pain}

One study made use of the Visual Analogue Scale (VAS) as a measurement scale to define 'postoperative pain'. The authors included patients with different types of hernia and concluded that $79 \%$ of lean patients $($ BMI $<30$ ) had a VAS of $<3$, compared with $71.9 \%$ of the obese patients (BMI $\geq 30$ ). This difference was statistically significant $(p=0.007)$. In this study, no distinction was made 
Table 1 Study characteristics

\begin{tabular}{|c|c|c|c|c|c|c|}
\hline Author & Study type & $\begin{array}{l}\text { Year of } \\
\text { publication }\end{array}$ & $\begin{array}{l}\text { Total number } \\
\text { of patients }\end{array}$ & $\begin{array}{l}\text { Level of } \\
\text { evidence }\end{array}$ & Type of hernia & Outcome measurements \\
\hline $\begin{array}{l}\text { Acevedo } \\
\text { and Léon }\end{array}$ & $\begin{array}{l}\text { Prospective } \\
\text { cohort study }\end{array}$ & 2010 & $\begin{array}{c}2031(326 \\
\text { umbilical } \\
\text { hernia) }\end{array}$ & $2 \mathrm{~B}$ & $\begin{array}{l}\text { Inguinofemoral, epigastric, } \\
\text { umbilical, incisional }\end{array}$ & Perioperative pain, complications \\
\hline $\begin{array}{l}\text { Bennett } \\
\text { et al. }\end{array}$ & $\begin{array}{l}\text { Prospective } \\
\text { cohort study }\end{array}$ & 2013 & 63 & $2 \mathrm{~B}$ & Paraumbilical & $\begin{array}{l}\text { Duration of surgery, patient } \\
\text { satisfaction }\end{array}$ \\
\hline $\begin{array}{l}\text { Dalenbäck } \\
\text { et al. }\end{array}$ & $\begin{array}{l}\text { Retrospective } \\
\text { cohort study }\end{array}$ & 2013 & 162 & $2 \mathrm{~B}$ & Umbilical & Recurrence, pain, complications \\
\hline $\begin{array}{l}\text { Garcia- } \\
\text { Urena } \\
\text { et al. }\end{array}$ & $\begin{array}{l}\text { Prospective } \\
\text { cohort study }\end{array}$ & 2000 & 157 & $2 \mathrm{~B}$ & Umbilical, epigastric & Complications, time to discharge \\
\hline $\begin{array}{l}\text { Kulacoglu } \\
\text { et al. }\end{array}$ & $\begin{array}{l}\text { Prospective } \\
\text { cohort study }\end{array}$ & 2012 & 100 & $2 \mathrm{~B}$ & Umbilical & $\begin{array}{l}\text { Pain (VAS), time to discharge, } \\
\text { complications, recurrences }\end{array}$ \\
\hline $\begin{array}{l}\text { Kurzer } \\
\text { et al. }\end{array}$ & $\begin{array}{l}\text { Prospective } \\
\text { cohort study }\end{array}$ & 2004 & 54 & $2 \mathrm{~B}$ & Umbilical & Pain, complications \\
\hline $\begin{array}{l}\text { Menon and } \\
\text { Brown }\end{array}$ & $\begin{array}{l}\text { Retrospective } \\
\text { cohort study }\end{array}$ & 2003 & 32 & $2 \mathrm{~B}$ & Umbilical & $\begin{array}{l}\text { Duration of surgery, complications, } \\
\text { recurrence }\end{array}$ \\
\hline $\begin{array}{l}\text { Sinha and } \\
\text { Keith }\end{array}$ & $\begin{array}{l}\text { Retrospective } \\
\text { cohort study }\end{array}$ & 2004 & 34 & 2B & Umbilical & $\begin{array}{l}\text { Duration of surgery, time to discharge, } \\
\text { complications, recurrences }\end{array}$ \\
\hline $\begin{array}{l}\text { Stabilini } \\
\text { et al. }\end{array}$ & $\begin{array}{l}\text { Prospective } \\
\text { cohort study }\end{array}$ & 2009 & 69 & $2 \mathrm{~B}$ & Umbilical, epigastric & $\begin{array}{l}\text { Time to discharge, recurrence, } \\
\text { complications }\end{array}$ \\
\hline
\end{tabular}

Table 2 Anesthesia techniques: the different types of anesthesia used and types of local anesthetics

\begin{tabular}{|c|c|c|c|}
\hline Author & LA & $\mathrm{LA}+$ sedation & LA or GA \\
\hline Acevedo and Léon & Lidocaine $0.5 \%$ & Not applicable & Not applicable \\
\hline Bennett et al. & Not applicable & Not applicable & $\begin{array}{l}\text { GA: not described } \\
\text { LA: xylocaine } 2 \% \text {, bupivacaine } 0.5 \%\end{array}$ \\
\hline Dalenback et al. & Not applicable & Not applicable & $\begin{array}{l}\text { GA: not described } \\
\text { LA: not described }\end{array}$ \\
\hline Garcia et al. & Not applicable & Lidocaine $1 \%$ + midazolam & Not applicable \\
\hline Kulacoglu et al. & Not applicable & Lidocaine, bupivacaine $0.5 \%+$ midazolam and fentanyl & Not applicable \\
\hline Kurzer et al. & Not applicable & Bupivacaine $0.25 \%+$ midazolam & Not applicable \\
\hline Menon and Brown & Not applicable & Xylocaine $1 \%$ + bupivacaine $0.5 \%$ + midazolam & Not applicable \\
\hline Sinha and Keith & Not applicable & Not applicable & $\begin{array}{l}\text { GA: not described } \\
\text { LA: xylocaine } 1 \%\end{array}$ \\
\hline Stabilini et al. & Not applicable & Not applicable & $\begin{array}{l}\text { GA: not described } \\
\text { LA: mepivacaine }\end{array}$ \\
\hline
\end{tabular}

between VAS scores per hernia type. It was neither described what VAS score patients had who were operated under local anesthesia [6].

Two other studies used terminology like 'mild, moderate or severe' and 'no severe postoperative pain' to report pain $[1,2]$. The authors did not mention which questionnaire or measurement scale was used for these statements.

\section{Duration of surgery}

Six authors investigated the duration of surgery, which ranged from 24 to $78 \mathrm{~min}[1,4,6,14,20,21]$. Table 3 shows that Bennett et al. were the only authors making a distinction between local anesthesia and general anesthesia for this outcome parameter. This study showed that the use of local anesthesia for paraumbilical hernia could lead to a shorter duration of surgery than the use of general anesthesia ( $p$ value $<0.0003$ ). However, patients with a lower BMI were more frequently operated under local anesthesia. When BMI was categorized to see if there was any difference between patients with a BMI less or more than 25 , and less or more than 30 (obese), there was no difference found in the length of the procedure. [14]. Kulacoglu et al. and Menon and Brown all included patients with umbilical 
Table 3 Duration of surgery

\begin{tabular}{lclll}
\hline Author & $N$ & Hernia type & Anesthesia & Duration of surgery, mean (min) \\
\hline Acevedo and Léon & 2031 & $\begin{array}{c}\text { Inguinofemoral, epigastric, } \\
\text { umbilical, incisional }\end{array}$ & LA & $\begin{array}{l}\text { Lean 62 }( \pm 8.6) \text { min } \\
\text { Obese 78 }( \pm 11.7) \text { min, } p<0.001\end{array}$ \\
Bennett et al. & 63 & Paraumbilical & LA + GA & LA 24 (17.5-30) \\
Garcia et al. & 157 & Umbilical, epigastric & & GA 35 (27-45), $p<0.0003$ \\
Kulacoglu et al. & 100 & Umbilical & LA & 49.7 \\
Menon and Brown & 32 & Umbilical & LA & $69(25-150)$ \\
Sinha and Keith & 34 & Umbilical & LA & $30(22-40)$
\end{tabular}

hernia treated with local anesthesia alone. Kulacoglu et al. showed that the mean operative time was 69 min (range 25-150 min), but in the patient group of Menon and Brown, the duration of surgery was significantly shorter with a mean operative time of $30 \mathrm{~min}$ (range $22-40 \mathrm{~min}$ ) $[1,20]$.

\section{Surgical site infection}

Surgical site infection (SSI) is a common postoperative complication and one of the most commonly described outcome parameters. The overall percentage of SSI was $3.4 \%(15 / 431)$, and ranged from $1 \%$ to $12.9 \%$ $[1,2,4,19,20]$. Three studies described that SSI responded well to conservative wound care or oral antibiotics, and no further treatment was required. Two remaining studies did not describe the treatment for SSI. Besides Acevedo and Léon, none of the authors described in which patient group SSI occurred [1, 2, 4, 19, 20]. Acevedo and Léon noted that there was a significantly higher rate of SSI in obese patients (BMI >30) than in non-obese patients, respectively, 2.1 and $0.7 \%(p<0.023)$. None of the articles specified the SSI rate per hernia or anesthesia type, nor was it described if SSIs were more frequently seen in patients treated with a mesh.

\section{Other postoperative complications}

The most frequent postoperative complications were seromas, with a range of $3-8.9 \%$, and an overall percentage of $4.8 \%$. All seromas either resolved spontaneously or were successfully treated with drainage $[1,3,4,21]$. The second most frequent postoperative complication were hematomas (1\%) $[1,3,4]$. There was one patient who suffered from postoperative bleeding and one other patient who suffered from intestinal obstruction. Both patients needed emergency surgery to resolve these complications [19]. Postoperatively, there were two patients suffering from allergic skin changes due to a plaster allergy [1]. Finally, there was one 86-year old patient operated under general anesthesia, who experienced episodes of confusion and dizziness postoperatively. Therefore, a prolonged hospital stay of 12 days was needed [21]. In total, 3 patients passed away after surgery, respectively, due to the following causes: liver cirrhosis, cerebral infarction and chronic renal failure. All causes were not related to the operation [3]. No perioperative complications were described. None of the articles made a comparison between type of anesthesia.

\section{Recurrence}

Seven studies described hernia recurrence rate as an outcome measurement [1-4, 19-21]. In three of these studies, no recurrences occurred $[1,2,20]$. The mean follow-up in these studies was 17 months (5-41), 43 months (28-67), and 70 months (27-142). The remaining four articles measured a recurrence rate ranging from 2 to $7.4 \%$ [3, 4, 19, 21]. These four studies did not mention which patients presented with a recurrence. Dalenbäck et al. were the only authors who included umbilical hernia patients alone. They made a distinction in recurrence rates between patients operated under general anesthesia and patients operated under local anesthesia. The authors found two recurrences (out of 144 patients) in the general anesthesia group and five recurrences (out of 144 patients) in the local anesthesia group. No statistical comparison was made between these two groups [19]. The studies did not describe how the recurrence was diagnosed: with physical examination only or with the addition of radiological examination.

\section{Duration of postoperative stay}

The mean duration of postoperative stay at the hospital varied from 2 hours to almost 2 days [1, 3, 4, 20, 21]. Table 4 gives an overview of the mean time before discharge. Kulacoglu et al. showed that patients with umbilical hernia, operated under local anesthesia, stayed $122 \pm 58 \mathrm{~min}$ in hospital before discharge [1]. Sinha and 
Table 4 Time to discharge

\begin{tabular}{llll}
\hline Author & Type of anesthesia & Type of hernia & Time to discharge (mean) \\
\hline Garcia et al. & Local anesthesia & Umbilical and epigastric hernia & $7.2 \mathrm{~h}$ \\
Kulacoglu et al. & Local anesthesia & Umbilical hernia & $122 \mathrm{~min} \pm 58 \mathrm{~min}(45-420)$ \\
Menon and Brown & Local anesthesia & Umbilical hernia & Same day, discharge before 20:00 p.m. \\
Sinha and Keith & General or local anesthesia & Paraumbilical hernia & LA: $89 \%$ discharged $<24 \mathrm{~h}$ \\
& & & GA: $47 \%$ discharged $<24 \mathrm{~h}$ \\
Stabilini et al. & General or local anesthesia & Umbilical hernia and epigastric hernia & 1.8 days $(3 \mathrm{~h}-15$ days $)$ \\
\hline
\end{tabular}

Keith described that $89 \%$ of the patients in the local anesthesia group were discharged in less than 24 hours, compared with $47 \%$ of the patients in the general anesthesia group [21]. The other articles did not specify the duration of stay for the type of anesthesia or type of hernia. The longest mean duration of stay was 1.8 days (range $3 \mathrm{~h}-15$ days) and was required due to severe associated diseases of the patients, emergency surgery for hernia strangulation and wound hematoma [3].

\section{Patient satisfaction}

Five studies reported on patient satisfaction, which was reported to be good in $89 \%$ till $97 \%$ of patients. Different methods of measuring this outcome parameter were used. Acevedo and Léon defined patient satisfaction as good, if the VAS for patient satisfaction was $>7$ points on a 10 points scale, in combination with a positive answer to the question 'would you recommend this kind of surgery to others?' This was measured at the 1 week control [6]. Sinha and Keith stated that $97 \%$ of their patient population was satisfied, according to the definition of Reitter [21]. The remaining three authors did not describe which questionnaire was used to define and measure patient satisfaction [1, 14, 19]. Two authors specified the patient satisfaction with regard to the body mass index of the patient [6, 14]. None of the articles specified the patient satisfaction per hernia type or anesthesia type $[1,19,21]$.

\section{Discussion}

The data from this systematic review reveal that the use of local anesthesia in umbilical hernia repair led to a shorter duration of postoperative stay, and that repair of a paraumbilical hernia performed under local anesthesia leads to a shorter duration of surgery. The use of local anesthesia did not lead to perioperative complications, serious postoperative complications, allergic responses or anesthesia-related deaths.
Umbilical hernia is a common surgical problem $[1,2]$. At this moment, data on umbilical hernia surgery under local anesthesia are only scarcely available. In contrast, groin hernias operated under local anesthesia are very well described in literature, and several studies have been performed [7, 10-13, 15, 22]. All these studies show the advantages of local anesthesia: less postoperative and general anesthesia-related complications, a shorter duration of surgery, less overnight admissions, less postoperative pain and no deaths. Van Veen et al. showed that significantly more urinary retentions occurred in patients undergoing Lichtenstein hernia repair under spinal anesthesia [7]. Furthermore, the conversion rate to general anesthesia was lower for patients operated under local anesthesia $(2 \%)$ than patients operated under spinal anesthesia (10\%) [15]. Nordin et al. also showed that local anesthesia has significant cost advantages compared to spinal anesthesia and general anesthesia [12]. We, therefore, performed a review of literature to investigate the safety and feasibility of the use of local anesthesia for umbilical hernia and to explore if there are any advantages to the use of local anesthesia for umbilical hernia.

We have performed a literature search and found no randomized controlled trials or other significant papers giving solid evidence for the use of local anesthesia as being superior in the treatment of umbilical hernias. Only a few small prospective or retrospective cohort studies were included in this review. The studies we included do not solely include umbilical hernias, and when the studies did include solely umbilical hernias, the authors did not describe their local anesthesia treatment well.

If we take a closer look at the included studies, a very high heterogeneity can be noticed. First of all; there is no consensus regarding the local anesthetic drug, and the technique to induce local anesthesia. The used local anesthetic drug varies from shorter acting lidocaine to the longer acting ropivacaine. The technique to inject is not discussed in most of the articles, one article mentioning the 'field block' as a way to induce local anesthesia. Some authors diluted their anesthetic with another type of anesthetic, others diluted it with saline or adrenaline. Amid 
et al. described a simple step-by-step infiltration technique for inguinal hernia, which is adapted and followed in most of the studies using local anesthesia for inguinal hernia [7, 10, 13, 15, 23]. Furthermore, Amid et al. used a solution which consisted of $1 \%$ lidocaine, $1 \%$ bupivacaine and epinephrine, which is used by other authors as well $[7,15]$. In local anesthesia of umbilical hernia, a standardized protocol is missing and should, therefore, be set up.

Pain is an important outcome measurement. However, not all studies describe perioperative or postoperative pain as an outcome measurement, and not all authors who do describe postoperative pain use the visual analog scale (VAS) to measure pain. Several studies regarding inguinal hernia have shown that postoperative pain in patients treated with local anesthesia is (significantly) lower compared to general anesthesia or spinal anesthesia [7, 15], but this outcome measurement is, despite of its importance, not thoroughly investigated for umbilical hernia. Due to this inconsistency, comparison of the studies is impossible.

Another essential outcome measurement is represented by postoperative complications. Surgical site infections and seromas are the most common complications. In the underlying studies, these complications either resolved spontaneously, were treated with drainage or antibiotics, and had no serious consequences for the patient. It is not clear if complications occurred more frequently among patients treated with local anesthesia, since the authors did not describe which patient developed a postoperative complication. There were no perioperative complications, nor any allergies against local anesthetics, or deaths described.

The hernia recurrence rate varied from 2 to $7.4 \%$, with a higher percentage for patients who were treated with primary sutures. This is comparable with the available literature, which describes a recurrence rate of approximately $2 \%$ for mesh repair, rising up to $8 \%$ for suture repair $[24,25]$. However, recently the cohort study of Christoffersen et al. showed that the total cumulated recurrence rate after primary repair was $10 \%$ for mesh repair and $21 \%$ for sutured repair after 55 months of follow-up ( $p=0.001)$ [26], which is a surprisingly high percentage. Dalenbäck et al. showed that the recurrence rate among umbilical hernia patients operated under local anesthesia was higher $(5 / 144)$ than in patients operated under general anesthesia (2/144). However, since there was no statistical comparison made, no conclusions can be drawn.

The duration of surgery varied from 24 to $78 \mathrm{~min}$ and was for all studies, with one exception, not specified per type of hernia or type of anesthesia. Bennett et al. were the only authors who did specify the outcomes per anesthesia type and showed that patients with a paraumbilical hernia operated under local anesthesia had a shorter duration of surgery than patients operated under general anesthesia. However, when BMI was categorized (more or less than BMI 25, and more or less than a BMI of 30), there were no differences found for duration of surgery. It can be concluded that BMI was a confounding factor, and patients who were operated under local anesthesia had more frequently a lower BMI.

Almost $90 \%$ of the patients operated with local anesthesia were discharged within 24 hours. This percentage rate is almost twice as high as patients operated under general anesthesia: 47\% was discharged within 24 hours. This is comparable with the available literature for groin hernias. Studies show a significantly shorter in hospital stay as well, and significantly less postoperative overnight admissions [7, 15]. There is no study comparing the difference in discharge time for local anesthesia and general anesthesia in umbilical hernia patients.

In our opinion, patients that would be eligible to undergo umbilical hernia repair under local anesthesia are cooperative patients with a low to normal BMI without certain mental disorders or physical disabilities and with a primary, up to maximally $4-5 \mathrm{~cm}$ large, non-recurrent umbilical hernia that will undergo repair in an elective setting. In case of end-stage cardiac and/or pulmonary disease local anesthesia must be considered as the preferred option.

This review has some limitations. Heterogeneity is the main disadvantage of this study. There is no consensus regarding the injection technique or the anesthetic drug that should be used. Postoperative pain, an essential outcome parameter, is not thoroughly described, and no standardized questionnaires were used to measure this outcome parameter. Furthermore, it is not clear if the complications and recurrences described in the included articles, occurred in the patient group we aim to investigate. Finally, we cannot conclude if patients with umbilical hernia treated with local anesthesia have a shorter duration of operation and a shorter duration of stay, since no comparison is made with a control group. Based on our findings, we cannot state that local anesthesia for umbilical hernia patients has any advantages.

\section{Conclusion}

Local anesthesia for umbilical hernia patients seems safe and feasible. However, the advantages of local anesthesia are not sufficiently demonstrated in the current available literature. Almost every outcome parameter is not specified for the patient group we aim to investigate: patients with umbilical hernia treated with local anesthesia. We still do not know if local anesthesia for umbilical hernia gives excellent results, so we cannot implement it in daily practice. Therefore, we propose to initiate a randomized controlled trial, comparing local anesthesia with general anesthesia for patients with umbilical hernia. This could reveal if local anesthesia has any advantages. 
Acknowledgements We would like to thank Wichor M. Bramer for his assistance in the literature search.

\section{Compliance with ethical standards}

Conflict of interest The authors of this manuscript have no conflicts of interest to disclose. AJ declares no conflict of interest. RK declares no conflict of interest. FM declares no conflict of interest. JJ declares no conflict of interest. JL declares no conflict of interest.

Ethical approval All procedures performed in studies involving human participants were in accodance with the ethical standards of the institutional and/or national research committee and with the 1964 Helsinki declaration and its later amendments or comparable ethical standards.

Human and animal rights This article does not contain any studies with animals performed by any of the authors.

Informed consent For this type of study formal consent is not required.

Open Access This article is distributed under the terms of the Creative Commons Attribution-NonCommercial 4.0 International License (http://creativecommons.org/licenses/by-nc/4.0/), which permits any noncommercial use, distribution, and reproduction in any medium, provided you give appropriate credit to the original author(s) and the source, provide a link to the Creative Commons license, and indicate if changes were made.

\section{Appendix 1}

\section{Literature search strategy}

\section{Pubmed Publisher 15}

In PubMed the following search strategy was performed: (((umbilic*[tiab] OR "abdominal wall" [tiab] OR ventral[tiab]) AND (herni*[tiab] OR defect*[tiab])) OR exomphal*[tiab])) AND ((((local[tiab] OR topical[tiab] OR region*[tiab] OR infiltrat*[tiab] OR conduct*[tiab] OR block*[tiab]) AND (anesthe*[tiab] OR anaesthe*[tiab])) OR ((ambula*[tiab] OR day[tiab] OR daycare[tiab] OR outpatient*[tiab] OR “short stay"[tiab]) AND (surg*[tiab] OR setting*[tiab] OR operati*[tiab] OR procedure*[tiab] OR treat*[tiab] OR therap*[tiab] OR repair*[tiab] OR hernioplast*[tiab] OR herniorrhaph*[tiab])) OR “day case"[tiab])) AND publisher[sb].

\section{Embase 507}

In Embase the following search strategy was performed: ('umbilical hernia'/de OR 'abdominal wall hernia'/de OR (umbilicus/de AND (hernioplasty/de OR herniorrhaphy/ de)) OR (((umbilic* OR 'abdominal wall' OR ventral) NEAR/6 (herni* OR defect*)) OR exomphal*):ab,ti) AND ('local anesthetic agent'/exp OR 'local anesthesia'/exp OR 'ambulatory surgery'/de OR 'outpatient department'/de OR outpatient/de OR 'ambulatory care'/de OR 'anesthetic needle'/de OR (((local OR topical OR region* OR infiltrat* OR conduct* OR block*) NEAR/3 (anesthe* OR anaesthe*)) OR ((ambula* OR day OR daycare OR outpatient* OR 'short stay') NEAR/3 (surg* OR setting* OR operati* OR procedure* OR treat* OR therap* OR repair* OR hernioplast* OR herniorrhaph*)) OR 'day case'):ab,ti).

\section{Medline 36}

In Medline the following search strategy was performed: ("Hernia, Umbilical"/OR "Hernia, Ventral"/OR (umbilicus/AND (herniorrhaphy/)) OR (((umbilic* OR "abdominal wall" OR ventral) ADJ6 (herni* OR defect*)) OR exomphal*).ab,ti.) AND ("Anesthesia, Local"/OR "Anesthetics, Local”/OR “Ambulatory Surgical Procedures"/OR "outpatients"/OR "Ambulatory Care"/OR (((local OR topical OR region* OR infiltrat* OR conduct* OR block*) ADJ3 (anesthe* OR anaesthe*)) OR ((ambula* OR day OR daycare OR outpatient* OR "short stay") ADJ3 (surg* OR setting* OR operati* OR procedure* OR treat* OR therap* OR repair* OR hernioplast* OR herniorrhaph*)) OR “day case").ab,ti.).

\section{Cochrane 6}

In Cochrane the following search strategy was performed: ((((umbilic* OR 'abdominal wall' OR ventral) NEAR/6 (herni* OR defect*)) OR exomphal*):ab,ti) AND ((((local OR topical OR region* OR infiltrat* OR conduct* OR block*) NEAR/3 (anesthe* OR anaesthe*)) OR ((ambula* OR day OR daycare OR outpatient* OR 'short stay') NEAR/3 (surg* OR setting* OR operati* OR procedure* OR treat* OR therap* OR repair* OR hernioplast* OR herniorrhaph*)) OR 'day case'):ab,ti).

\section{Web of Science 152}

In Web of Science the following search strategy was performed TS $=(((()$ umbilic* OR "abdominal wall” OR ventral) NEAR/6 (herni* OR defect*)) OR exomphal*)) AND ((()local OR topical OR region* OR infiltrat* OR conduct* OR block*) NEAR/3 (anesthe* OR anaesthe*)) OR ((ambula* OR day OR daycare OR outpatient* OR "short stay") NEAR/3 (surg* OR setting* OR operati* OR procedure* OR treat* OR therap* OR repair* OR hernioplast* OR herniorrhaph*)) OR “day case”))).

\section{Scopus 230}

In Scopus the following search strategy was performed TITLE-ABS-KEY((()(umbilic* OR “abdominal wall” OR 
ventral) W/6 (herni* OR defect*)) OR exomphal*)) AND ((()local OR topical OR region* OR infiltrat* OR conduct* OR block*) W/3 (anesthe* OR anaesthe*)) OR ((ambula* OR day OR daycare OR outpatient* OR "short stay") W/3 (surg* OR setting* OR operati* OR procedure* OR treat* OR therap* OR repair* OR hernioplast* OR herniorrhaph*)) OR “day case”))).

\section{References}

1. Kulacoglu H, Yazicioglu D, Ozyaylali I (2012) Prosthetic repair of umbilical hernias in adults with local anesthesia in a day-case setting: a comprehensive report from a specialized hernia center. Hernia 16(2):163-170

2. Kurzer M, Belsham PA, Kark AE (2004) Tension-free mesh repair of umbilical hernia as a day case using local anaesthesia. Hernia 8(2):104-147

3. Stabilini C, Stella M, Frascio M et al (2009) Mesh versus direct suture for the repair of umbilical and epigastric hernias. Ten-year experience. Ann Ital Chir 80(3):183-187

4. Garcia-Urena MA, Garcia MV, Ruiz VV et al (2000) Anesthesia and surgical repair of aponeurotic hernias in ambulatory surgery. Ambul Surg 8(4):175-178

5. Muysoms F, Campanelli G, Champault GG et al (2012) EuraHS: the development of an international online platform for registration and outcome measurement of ventral abdominal wall hernia repair. Hernia 16(3):239-250

6. Acevedo A, Leon J (2010) Ambulatory hernia surgery under local anesthesia is feasible and safe in obese patients. Hernia 14(1):57-62

7. Nordin P, Zetterstrom H, Gunnarsson U et al (2003) Local, regional, or general anaesthesia in groin hernia repair: multicentre randomised trial. Lancet 362(9387):853-858

8. Callesen T, Bech K, Kehlet H (2001) One-thousand consecutive inguinal hernia repairs under unmonitored local anesthesia. Anesth Analg 93(6):1373-1376

9. Kark AE, Kurzer MN, Belsham PA (1998) Three thousand one hundred seventy-five primary inguinal hernia repairs: advantages of ambulatory open mesh repair using local anesthesia. J Am Coll Surg 186(4):447-455

10. Amid PK, Shulman AG, Lichtenstein IL (1994) Local anesthesia for inguinal hernia repair step-by-step procedure. Ann Surg 220(6):735-737

11. Teasdale C, McCrum AM, Williams NB et al (1982) A randomised controlled trial to compare local with general anaesthesia for short-stay inguinal hernia repair. Ann R Coll Surg Engl 64(4):238-242

12. Gonullu NN, Cubukcu A, Alponat A (2002) Comparison of local and general anesthesia in tension-free (Lichtenstein) hernioplasty: a prospective randomized trial. Hernia 6(1):29-32

13. van Veen RN, Mahabier C, Dawson I et al (2008) Spinal or local anesthesia in lichtenstein hernia repair: a randomized controlled trial. Ann Surg 247(3):428-433

14. Bennett PC, Kumar B, Coveney EC (2013) Local anaesthetic repair of uncomplicated paraumbilical Hernia without sedation: peri-operative pain and patient satisfaction. Hernia 17(4):499-504

15. Nordin P, Zetterstrom H, Carlsson P et al (2007) Cost-effectiveness analysis of local, regional and general anaesthesia for inguinal hernia repair using data from a randomized clinical trial. Br J Surg 94(4):500-505

16. Callesen $T$ et al (2003) Inguinal hernia repair: anaesthesia, pain and convalescence. Dan Med Bull 50(3):203-218

17. Liberati A, Altman DG, Tetzlaff J et al (2009) The PRISMA statement for reporting systematic reviews and meta-analyses of studies that evaluate health care interventions: explanation and elaboration. J Clin Epidemiol 62(10):e1-e34

18. http://www.cebm.net/ocebm-levels-of-evidence/

19. Dalenback J, Andersson C, Ribokas D et al (2013) Long-term follow-up after elective adult umbilical hernia repair: low recurrence rates also after non-mesh repairs 17(4):493-497

20. Menon VS, Brown TH (2003) Umbilical hernia in adults: day case local anaesthetic repair. J Postgrad Med 49(2):132-133

21. Sinha SN, Keith T (2004) Mesh plug repair for paraumbilical hernia. Surgeon 2(2):99-102

22. de Craen AJ, van Vliet HA, Helmerhorst FM (2005) An analysis of systematic reviews indicated low incorporation of results from clinical trial quality assessment. J Clin Epidemiol 58(3):311-313

23. Amid PK, Shulman AG, Lichtenstein IL (1994) [A five-step technique for local anesthesia in inguinal hernia repair] Eine Technik in funf Schritten zur Lokalanaesthesie bei der Leistenhernienreparation. CHIRURG 65(4):388-390

24. Nguyen MT, Berger RL, Hicks SC et al (2014) Comparison of outcomes of synthetic mesh vs suture repair of elective primary ventral herniorrhaphy: a systematic review and meta-analysis. JAMA Surg 149(5):415-421

25. Aslani N, Brown CJ (2010) Does mesh offer an advantage over tissue in the open repair of umbilical hernias? A systematic review and meta-analysis. Hernia 14(5):455-462

26. Christoffersen MW, Helgstrand F, Rosenberg J et al (2014) Longterm recurrence and chronic pain after repair for small umbilical or epigastric hernias: a regional cohort study. Am J Surg 209(4):732-752 